\title{
DE QUÉ HABLAMOS CUANDO HABLAMOS DE POLÍTICAS DE INFORMACIÓN
}

\author{
Lluís Anglada
}

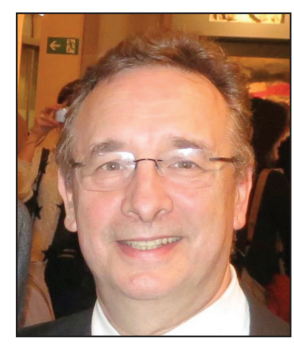

Lluís Anglada es director del Área de Biblioteques, Informació i Documentació del Consorci de Serveis Universitaris de Catalunya (CSUC) después de ser el director del Consorci de Biblioteques Universitàries de Catalunya (CBUC) de 1997 a 2013. Anteriormente fue director de las bibliotecas de la Universitat Politècnica de Catalunya y profesor de la Escola Universitària Jordi Rubió i Balaguer de Biblioteconomia i Documentació de la Universitat de Barcelona. Es licenciado en filosofía y diplomado en biblioteconomía y documentación. Ha sido miembro del Global Council de OCLC y actualmente pertenece a la Comisión Ejecutiva de Liber. Es autor y promotor de los blogs Bdig y Blok de Bid. Imparte regularmente talleres y conferencias y publica artículos sobre bibliotecas y consorcios bibliotecarios.

http://orcid.org/0000-0002-6384-4927

Consorci de Serveis Universitaris de Catalunya Gran Capità, 2-4, edif. Nexus, 4ํㅡ pl., 08034 Barcelona, España langlada@gmail.com

\begin{abstract}
Resumen
Una política de información es una acción concertada de varios agentes con la información como objeto y con determinadas finalidades como metas. La crisis que vivimos no sólo es económica sino también tecnológica y de cambio de modelo organizativo, y está mostrando ya algunos efectos de la desregulación y la no planificación. Sólo podremos establecer políticas solidas de información si encontramos un conjunto de acciones alrededor de las cuales se puedan alinear sin fricciones un amplio espectro de agentes. 1. En el mundo de lo impreso deberán conseguir que se pudiera conocer de forma fácil todo lo que hay, que fuera accesible y que lo que se usa poco y tenderá a usarse menos se conserve. 2. Las instituciones patrocinadoras de la investigación pueden y deben ponerse de acuerdo en facilitar el acceso abierto a la información y a los datos. 3. Finalmente, la biblioteca organización ha de poder prestar libros digitales, ofrecer información digital para el uso de todos y garantizar que el acervo de lo nacido digital pasará a las generaciones futuras. A esto se le deben añadir planes para conseguir la formación para la información en las escuelas. En estos momentos de cambio, rodeados de incertidumbres y con algunas debilidades notables, parte de nuestros deberes profesionales deben dedicarse al tejido de acuerdos que permitan actuar coordinadamente en direcciones determinadas.
\end{abstract}

\section{Palabras clave}

Políticas de información, Política científica, Planificación, Coordinación, Cooperación, Transformación social, Crisis económica, Planes de futuro, Bibliotecas, Acceso abierto, Preservación, Acceso universal.

Title: What do we mean when we talk about information policies

\section{Abstract}

An information policy is a concerted action of various agents with information as the object, having as a purpose certain goals. The crisis we face is not only economic but also technological and due to changes in the organizational model, and we are already seeing some effects of deregulation and no planning. We can only establish solid information policies if we find a set of actions around which a wide spectrum of agents can be aligned without friction. 1. In the print world, policies should allow us to determine what exists and make that easily accessible, and know what is little used and will tend to be used less and ensure that it is preserved. 2. Research funding institutions can and must agree to facilitate open access to information and data. 3. Finally, the library-organization must be able to provide digital books, offer digital information that anybody can use, and ensure that born-digital materials are passed on to future generations. To this we must add the need to plan for information literacy training in schools. In these times of change, surrounded by uncertainty and some notable weaknesses, part of our professional duties should be devoted to elaborate agreements enabling us to take coordinated action in certain directions.

\section{Keywords}

Information policy, Science policy, Planning, Coordination, Cooperation, Social transformation, Economic crisis, Future plans, Libraries, Open access, Preservation, Universal access. 
Anglada, Lluís (2014). “De qué hablamos cuando hablamos de políticas de información". El profesional de la información, marzo-abril, v. 23, n. 2, pp. 105-111.

http://dx.doi.org/10.3145/epi.2014.mar.01

\section{Introducción}

(Uno que ya tiene su edad recuerda que) en 1983 unas históricas Primeres jornades de teledocumentació a Catalunya concluyeron que era necesario "elaborar un programa general de información para Catalunya que analice las necesidades de los usuarios tanto del sector público como del sector privado, defina las coberturas adecuadas para satisfacerlas, identifique los medios personales, tecnológicos y organizativos necesarios y periodifique debidamente la consecución de los objetivos que se establezcan"1.

Podemos encontrar otras definiciones de lo que son políticas de la información pero ésta nos vale, ya que además se formuló en un momento en el que el tema tenía plena vigencia a nivel internacional. No en vano la Unesco había iniciado un programa general de información (PGI) que bajo el nombre de Unisist pretendía "coordinar las tendencias existentes hacia la cooperación y actuar como catalizador para los desarrollos necesarios en información científica" y tenía como finalidad "el establecimiento de una red flexible de servicios y sistemas de información basado en la cooperación voluntaria" ${ }^{2}$

En un mundo con preponderancia de lo digital se entenderá cada vez menos que las bibliotecas no puedan informar de forma exhaustiva sobre los bienes públicos impresos y proporcionar acceso a los mismos

Porque, al final, una política de información no es más que la acción concertada de diferentes agentes con la información como objeto y con determinadas finalidades como metas. La euforia tecnológica y de recursos de la década anterior y la posterior del cambio de siglo crearon la sensación de que con la información todo era posible y minimizaron la necesidad de coordinarse para conseguir determinados fines. Los enormes cambios que ha supuesto la información digital y en red para los sistemas de información han comportado mejoras evidentes, pero también un cierto desconcierto sobre las direcciones que tomar y -sobre todo- un cierto optimismo sobre la no necesidad de tomar direcciones de forma conjunta ya que, al final, la tecnología por sí sola conseguiría lo que no podía conseguir la acción coordinada de las personas.

La crisis que vivimos no es sólo económica sino también tecnológica y de cambio de modelo organizativo, y está mostrando ya algunos efectos de la desregulación y la no planificación. El acceso a la información por parte de la totalidad de los ciudadanos, la existencia de información sin interés comercial y la preservación para el futuro de la información digital no se conseguirán de forma espontánea y sin esfuerzo. En los últimos años, el movimiento del acceso abierto ha mostrado también que la gran fuerza de una acción concertada en una dirección dada tiene el poder de modificar maneras de hacer centenarias. Pero las tendencias (lógicas) por parte de las empresas editoriales de continuar reteniendo derechos sobre la información y los datos han mostrado también que las solas fuerzas del mercado unidas a la ausencia de políticas de la información tienen efectos restrictivos sobre este bien público que es la información.

Si esto fuera así (y yo así lo creo) tener o no políticas de información dependería menos de los recursos existentes (normativos o de infraestructura) que de la capacidad de las instituciones que manejan información de llegar a acuerdos sobre cuáles deben ser estas acciones concertadas y sobre el papel de cada uno en emprenderlas. Las políticas de información exigen también tener estrategias definidas, ¿deben ser globales o sectoriales? ¿generadas por el Estado o por las bibliotecas? ¿dirigidas o participativas? ¿de instituciones de un mismo tipo o de un amplio espectro? De buen seguro que existen distintas respuestas igualmente válidas a estas preguntas, y que elegir la mejor estrategia depende de variables de tiempo y lugar, pero a mi parecer sólo podemos establecer políticas sólidas de información si encontramos un conjunto de acciones alrededor de las cuales se pueda alinear sin fricciones un amplio espectro de agentes.

Hay tres universos que deberían permitir coincidencias amplias: el mundo de lo impreso, el de la investigación y el de la cultura. Las posibilidades de establecer políticas de información en estos ámbitos se basan en consensos generalizados sobre lo que se debería o sería bueno hacer en cada uno de ellos.

\section{Políticas de información para el reino de lo impreso}

En el ecosistema de la información, la especie de los documentos impresos está en un proceso de retroceso y mutación. Su importancia será progresivamente menguante en las próximas décadas dentro de las cuales los expertos no prevén su desaparición. Las bibliotecas como instituciones coleccionadoras y gestoras de lo impreso deberán conseguir que se pueda conocer de forma fácil todo lo que hay, que fuera accesible y que lo que se usa poco y tenderá a usarse menos se conserve.

Los dos objetivos primeros fueron ya objeto de políticas de información de la IFLA con el programa de control bibliográfico universal $(C B U)$ y el de disponibilidad universal de las publicaciones (UAP). Ninguno de los dos es nuevo en el entorno de la biblioteconomía y la documentación, pero los dos deberán ser realizables en un corto espacio de tiempo. Tener localizados todos los documentos impresos de todas las bibliotecas parece un objetivo razonable y conseguible. 
Worldcat de OCLC se aproxima a ello desde una perspectiva tan global que quizá sea excesiva a efectos prácticos, pero la verdad es que tener al menos dos catálogos colectivos para las bibliotecas españolas (uno para bibliotecas universitarias, especializadas y nacionales, y otro para públicas y escolares) sería útil y nada difícil.

En un mundo con preponderancia de lo digital se entenderá cada vez menos que las bibliotecas no puedan informar de forma exhaustiva sobre los bienes públicos impresos y proporcionar acceso a los mismos. Lo que hoy llamamos préstamo interbibliotecario debería ser un conjunto concatenado de acuerdos entre redes y sistemas bibliotecarios que proporcionara de forma transparente y rápida cualquier documento que un usuario necesite. $Y$ esto continuará siendo necesario, porque aunque tendremos acceso a mucha información en formato digital, una buena parte de la misma no lo estará por carecer de suficiente interés comercial para digitalizarla o por existir obstáculos legales para hacerlo.

La ciencia y la innovación producen beneficios económicos y mejoran el bienestar social

En estos momentos las bibliotecas españolas custodian 175 millones de volúmenes impresos ${ }^{3}$, su prescindibilidad irá en aumento, pero no la necesidad de mantener suficientes copias de cada título. Los interesantes estudios de OCLC sobre solapamiento de colecciones impresas y digitales ${ }^{4}$ muestran que el acceso a la totalidad de la información impresa generada por la humanidad va a necesitar depósitos donde almacenar de forma barata y coordinada los múltiples libros y revistas que tendrán un uso demasiado bajo como para estar en los estantes de las bibliotecas y que no hayan encontrado motivo de pasar a la Red.

El dominio en extinción de lo impreso requiere medidas de preservación que la tecnología hace posibles. Una red federada de catálogos colectivos y de almacenes de impresos debería permitir que, a través de acuerdos de préstamo entre los sistemas bibliotecarios, las colecciones bibliotecarias impresas fueran, a efectos prácticos, un todo.

\section{Políticas de información para la ciencia}

De forma similar el entorno de la información científica genera hoy consensos alrededor de los cuales se pueden articular políticas de la información. La idea central ha sido expresada de forma clara y directa por el Finch report ${ }^{5}$ : la ciencia y la innovación producen beneficios económicos y de mejora del bienestar social y se espera que una ciencia abierta genere más y mejor ciencia. Así las instituciones patrocinadoras de la investigación (las bibliotecas entre ellas) pueden fácilmente ponerse de acuerdo en facilitar el acceso abierto a la información y a los datos.

Pero, si para el caso de lo impreso se daba coincidencia amplia sobre los objetivos finales y sobre la manera de conseguirlos, para el caso de la información científica el acuerdo es mucho más fuerte en las metas que en los caminos para conseguirlas. Y esta diferencia no es banal.
El Open Access (OA) tiene poco más de diez años. La idea, que surgió alrededor de una carta abierta firmada por algunos científicos, ha pasado de ser un ideal romántico a un horizonte tangible. Las cifras sobre el porcentaje de artículos científicos en abierto varían, aunque en 2009 Björk et al. ${ }^{6}$ las estimaron ya en un $20 \%$. A pesar de ello, en estos momentos nadie duda (tampoco los editores comerciales) de que el acceso abierto a las revistas o a los artículos va a ser la forma dominante de la comunicación científica a corto plazo (digamos el año 2020). La meta está pues clara, pero los caminos divergen en los colores (vías verde y dorada, con variantes) y contenedores (repositorios o revistas). Pero la visión de resultado final permite construir políticas de información favorecedoras del OA siempre que éstas se mantengan abiertas a las diferentes maneras de conseguir el acceso abierto para el lector y combinen mandatos con incentivos y facilidades.

Con el acceso abierto a los datos pasa algo parecido. La meta de ofrecerlos en abierto es compartida extensamente, pero aquí la divergencia no está en los caminos para conseguirlo sino en la incertidumbre de una ruta aún no trazada. Una publicación reciente sobre el tema tiene un título significativo: "Starting the conversation: university-wide research data management policy"7. Como en otras ocasiones en la historia reciente (la tele-documentación, el OA o las compras consorciadas) las bibliotecas no tienen por qué ser los agentes de la gestión y almacenamiento de los datos producidos por la investigación científica, pero son los agentes mejor situados para ejercer de catalizadores. Y esta posición de liderazgo en un entorno incierto podría permitir también alianzas amplias sobre las que construir políticas de la información (en este caso, datos).

Las bibliotecas no tienen por qué ser los agentes de la gestión y almacenamiento de los datos producidos por la investigación científica, pero son los agentes mejor situados para ejercer de catalizadores

Creo también que hay consenso en considerar que la compra conjunta de recursos digitales (consorcios) o la compartición de infraestructuras (repositorios y portales) son caminos que refuerzan la capacidad de acción de las bibliotecas. Como en el OA y para con los datos, no existe consenso sobre el camino a seguir. Hay consorcios especializados en una función y multi-tipo, ámbitos de compras estatales y regionales, tutelados por el estado o cooperativas de base... $Y$ también diversas opciones en lo relativo a infraestructuras (repositorios institucionales o temáticos, por ejemplo). Pero, en cualquier caso, la cooperación y la formación de alianzas parecen un eje de futuro que debería formar parte de una política de información en el área de la investigación.

El nuevo paradigma en construcción para la comunicación científica es un campo abierto a nuevas soluciones, un terreno para la experimentación. Pero la exploración de terrenos desconocidos es mejor hacerla acompañados. Las direcciones están trazadas pero los caminos son poco transitables. 


\section{PLAN ESTRATEGICO DE REBIUN 2020}

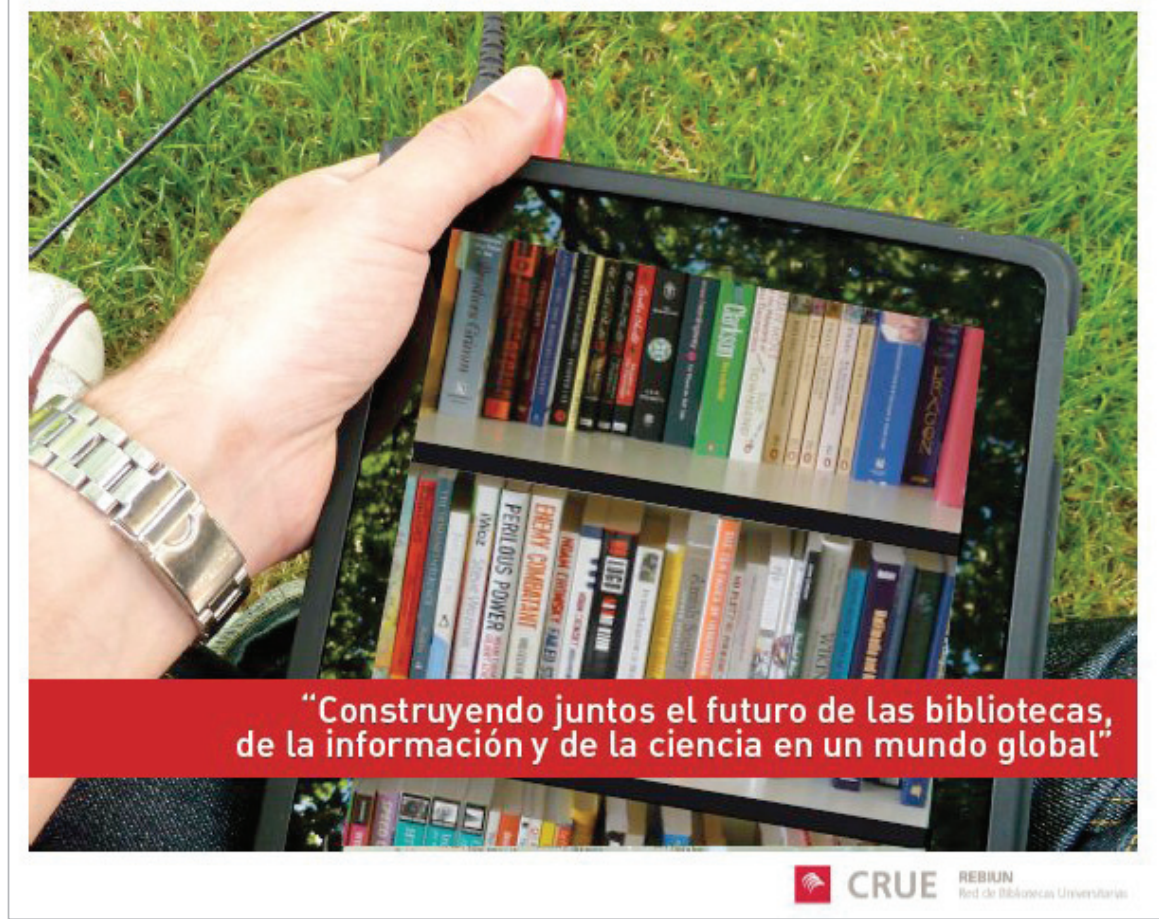

http://www.mcu.es/bibliotecas/docs/MC/ConsejoCb/CTC/Bib_Univ/Planestrategico2020.pdf mercado vigentes.

No se puede banalizar el impacto del préstamo de libros en formato digital sobre la sostenibilidad económica de las empresas editoriales y las cadenas de distribución de la cultura. Las editoriales han contribuido a la difusión de la cultura a la vez que han sido empresas con finalidad de lucro e incluso a veces muy rentables. El fomento de la lectura y las funciones educativas y sociales realizadas a través del préstamo gratuito de libros por parte de las bibliotecas debe poder continuar haciéndose en beneficio del ciudadano y de la sociedad y debe hacerse manteniendo algún tipo de negocio para el editor. Carme Fenoll definía el problema de esta manera: "los editores no acaban de dar luz verde al préstamo de libros electrónicos en las bibliotecas públicas porque creen que éste desequilibraría el sector y repercutiría disminuyendo sus ventas" ${ }^{\prime \prime}$. Lo cierto es que -a diferencia del caso de las revistas científicas con el pago por publicar- en la edición comercial no se ha encontrado aún el equili-

Aquí es perfectamente posible establecer unas políticas de información amplias siempre que sus agentes sean flexibles y no definan programas de actuación demasiado escorados hacia alguna de las opciones en juego.

En la edición comercial no se ha encontrado aún el equilibrio que satisfaga a bibliotecas y editores

\section{Políticas de información para la lectura y la cultura}

El dominio emergente de lo digital reconfigurará las relaciones del ciudadano con la cultura y para éste debe suponer más información y más capacidad de usarla. Los espacios de uso libre de la información dentro de lo que han sido las bibliotecas hasta ahora deben extenderse al espacio virtual donde el ciudadano ha de poder encontrar la información que rellena su ocio, le forma y configura su memoria. La biblioteca no edificio y sí organización ha de poder prestar libros digitales, ofrecer información digital para el uso de todos y garantizar que el acervo de lo nacido digital pase a las generaciones futuras.

Para los universos anteriores hemos afirmado que se daba coincidencia amplia sobre los objetivos finales. Sin embargo, en éste, tales objetivos pueden incluso estar en entredicho ya que la información digital erosiona los modelos de brio que satisfaga a las dos partes. La solución no es ni será fácil ni tampoco será local. Debería crearse una alianza de autores, editores, libreros y bibliotecarios que intentara definir un acuerdo nacional para el préstamo digital. Un acuerdo que sería provisional y que probablemente sólo pueda configurarse de momento de forma piloto. Al mismo tiempo esta alianza debería tener la vista puesta en las operaciones parecidas emprendidas por países próximos ${ }^{9}$.

El segundo elemento de una política de información para la ciudadanía sería el fomento de la digitalización de documentos no nacidos digitales y sobre todo su puesta a disposición en formatos interoperables y regímenes de uso amplios. Lo primero parece formar parte de lo asumido por todos y podríamos citar aquí diferentes iniciativas ejemplares de digitalización de documentos. Creo que los consensos y planes de acción institucionales deben centrarse en la interoperabilidad de los documentos digitalizados (muchos de ellos con recursos públicos). El enorme universo de la cultura humana se ha desplegado en nichos que tienen más que ver con la forma que han tomado los documentos que con sus contenidos. El mundo de lo digital nos permitirá recomponer los nichos en un todo, al menos en el dominio del acceso. Para que una persona pueda acceder a los contenidos que le interesan de forma independiente de la forma que éstos tomen (o de las instituciones que los almacenen), los metadatos deben ser interutilizables por aplicaciones indistintamente de la institución en la que se hayan producido. Probablemente la web semántica y los datos abiertos y enlazados (linked open data) sean la solución, pero antes 
de un uso extenso de los mismos, los diferentes colectivos profesionales que tratan con documentos culturales deberán hacer esfuerzos de aproximación en la forma como los tratan.

Finalmente la preservación de lo nacido digital deberá afrontarse también en alianza entre diferentes sectores. No serán las instituciones culturales las que posean la infraestructura y la tecnología que se requerirá para preservar la cada vez mayor

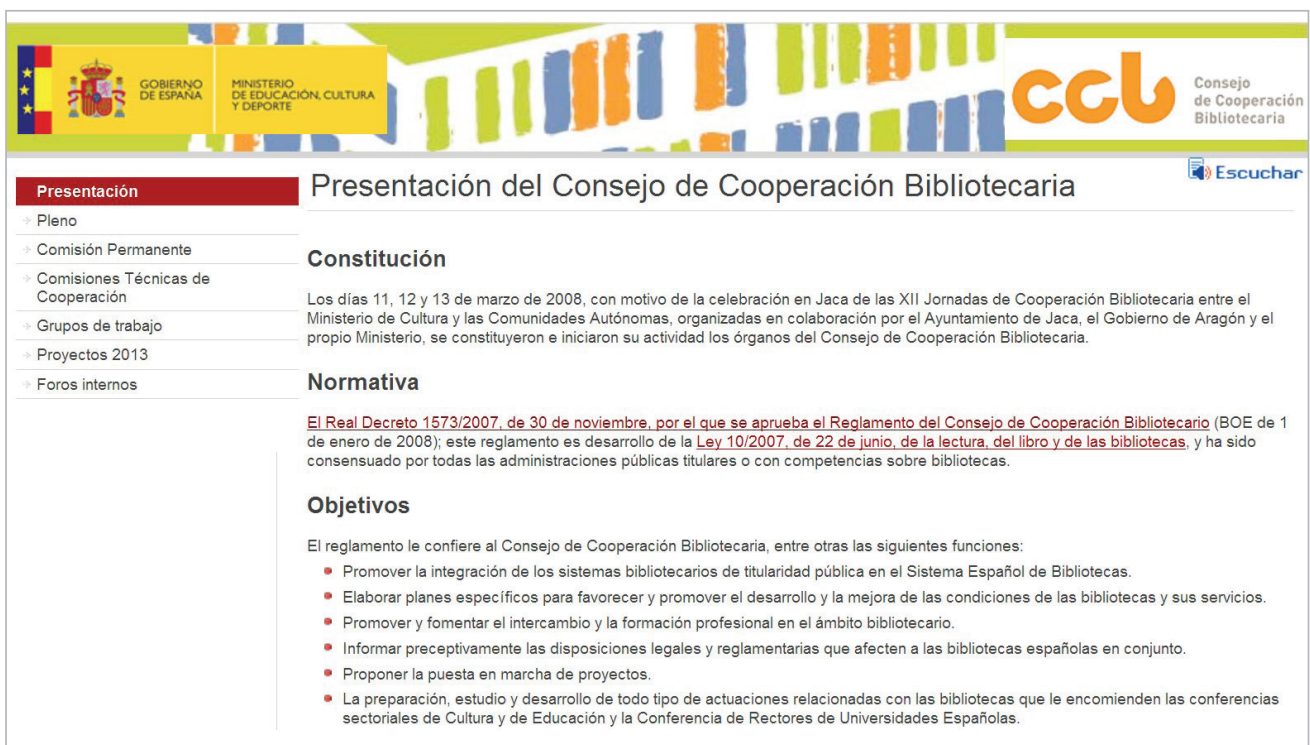

http://www.mcu.es/bibliotecas/MC/ConsejoCB/Presentacion.html cantidad de información nacida digital que deberíamos garantizar que se conservara para el futuro. Ni serán tampoco los tecnólogos quienes tendrán los conocimientos y la sensibilidad que se necesitarán para manejar documentos digitales de diferente procedencia y contexto. Ni hay consenso sobre lo que debemos preservar ni instrumentos preparados para hacerlo. Y en este caso la solución tampoco podrá proceder de una sola instancia.

En este campo de información para la ciudadanía los agentes se multiplican: autores, editores y libreros; archivos, bibliotecas y museos; documentalistas e informáticos... En todos los casos se trata de establecer alianzas con sectores que han sido vecinos en el reino de lo impreso, pero vecinos incomunicados. Las políticas de la información que se deben establecer aquí quizá sean las más difíciles de tejer debido a la heterogeneidad de culturas corporativas e intereses de sus agentes.

\section{El caso de las bibliotecas escolares}

Hemos dibujado tres ámbitos relativamente autónomos de construcción de acciones concertadas o de políticas de la información: uno alrededor del libro impreso, otro alrededor de la investigación y un tercero alrededor de la cultura. Pero hay uno que debería conciliar los acuerdos de los tres y sumar aún a nuevos agentes: las bibliotecas escolares.

Algunas mentes lúcidas y algunos gobiernos creen que la sostenibilidad y desarrollo de nuestra sociedad pasan por las innovaciones que pueda aportar la ciencia y que ésta dará más y mejores resultados si se realiza en abierto. Esto supone un impulso importante de políticas de información, pero deberíamos saber aprovechar este empujón para extender las acciones. La innovación puede mejorar si la información-objeto se hace más accesible, pero lo hará también, si y solo si, los científicos, profesionales y ciudadanos pueden integrar la información de forma creativa y crítica.

Esto solo se conseguirá con una formación para la información, formación que debe iniciarse en la escuela y que debe apoyarse en planes y profesionales especializados. ¿Alguien

puede imaginase una educación física sin instalaciones deportivas y sin profesorado preparado? Menos deberíamos podernos imaginar una educación para la información sin bibliotecas escolares y sin profesionales preparados para atenderlas.

Seguramente Rebiun sea el mejor ejemplo en España de la capacidad de las bibliotecas asociadas para conducir nuestro futuro

\section{Quién y cómo}

¿Quién debería establecer una política de información, es decir aquello que hemos definido como una acción concertada de diferentes agentes con la información como objeto y con determinadas finalidades como metas? Creo que desde la 'vieja Europa' tenemos cierta tendencia a considerar que debería hacerlo el Estado, pero la administración ${ }^{10}$ se ha mostrado conservadora y remisa a tomar un papel de liderazgo en esta época de cambios profundos. En el caso español esto se agudiza por la falta de tradición planificadora, interés por la cultura y fragmentación de competencias.

Francis Fukuyama afirma que "una única característica cultural aglutinante condiciona el bienestar de una nación, así como su capacidad para competir: el nivel de confianza inherente a la sociedad"11 y que ésta se mide por el dinamismo de su sociedad civil y la cuantía del capital social que acumulan. La capacidad de establecer alianzas y asociaciones de intereses configura el desarrollo de una sociedad y, siguiendo esta argumentación, deberíamos dejar en manos de los agentes la concertación de acciones que configuran una política de información.

Si las metas que hemos presentado como potencialmente comunes realmente lo son, el camino para conseguirlas de- 
bería ser la adición de agentes a su alrededor, es decir, a través de las asociaciones profesionales y del establecimiento de alianzas entre diferentes agentes. De hecho, sólo el mismo ejercicio de la asociación puede mostrarnos cuáles son las acciones concretas que concilian consenso y que se revelan como determinantes para configurar determinado futuro.

No hay certeza sobre el devenir, pero sí hay valores a defender y objetivos a perseguir. Hay consensos posibles en la diversidad de tipos de instituciones e intereses comunes por encima de los propios de un sector o una organización. Quizá siempre, pero más en este momento en que la tecnología y el mercado están reconfigurando una sociedad diferente, los puntos de vista sectoriales sólo prevalecerán si son expuestos y defendidos por el sector que los representa. El acceso generalizado a la información por parte de una ciudadanía capaz de utilizarla para su crecimiento personal y colectivo no será un bien que se consiga sin explorar organizadamente las alternativas existentes y sin apostar dentro de la incertidumbre por las que se considere más adecuadas.

\section{En el campo de la información para la ciudadanía las políticas de la informa- ción quizá sean las más difíciles de tejer debido a la heterogeneidad de culturas corporativas e intereses}

Esto defendiendo -tal como se hace evidente- el reforzamiento del asociacionismo profesional y el papel de las asociaciones profesionales e institucionales en el establecimiento de políticas de información. En un mundo globalizado no se encontrarán caminos para la preservación de lo nacido digital o del préstamo de libros electrónicos en las bibliotecas, por ejemplo, desde lo local. Esto, de forma evidente, no excluye la experimentación a nivel institucional o territorial, pero el campo de juego es mucho más grande, es multitipo, es intersectorial y es de alcance estatal y mundial.

Seguramente Rebiun, la asociación de bibliotecas universitarias, sea el mejor ejemplo en España de la capacidad de las bibliotecas asociadas para conducir nuestro futuro. Sus planes estratégicos, con mayores o menores aciertos en cada momento, pero continuados en el tiempo han conseguido no sólo cambios notables sino además visibilizarse como agentes de cambio ${ }^{12}$. La acción concertada de varios ha fortalecido al grupo, sí, pero sobre todo ha fortalecido a las partes.

Otro ejemplo destacable -este a nivel europeo- es el esfuerzo que actualmente está haciendo Liber para reposicionar las bibliotecas nacionales, universitarias y de investigación en este entorno cambiante. Las líneas de trabajo han derivado de un trabajo previo definiendo una visión estratégica del que han derivado tres líneas de acciones que Liber propone a sus miembros: redefinir la biblioteca física, posicionar la biblioteca como un socio activo en la investigación científica y actuar de forma conjunta frente la Unión Europea.
De una forma parecida, es decir elaborando una visión estratégica de la que se deriva un plan de acción, está actuando el Consejo de Cooperación Bibliotecaria. Éste aprobó su primer plan estratégico para el período 2013-15, compuesto de tres líneas: promoción y fomento de las bibliotecas, sostenibilidad de los servicios bibliotecarios en el nuevo entorno informacional y social, y evolución del funcionamiento del Consejo ${ }^{13}$. El Plan del CCB generó un grupo de trabajo que ha hecho una labor de prospectiva que destaca las diez áreas que más van a cambiar en nuestras bibliotecas en los próximos años y del que se derivan propuestas de trabajo conjuntas ${ }^{14}$. De forma muy sintética, el estudio de tendencias nos indica tres direcciones:

- la biblioteca debe ser creadora y educadora de comunidades;

- los edificios serán espacios sociales, y

- sus servicios se adaptarán a la realidad digital.

Y dos caminos:

- flexibilizar las estructuras y procesos de las bibliotecas; e - incrementar la cooperación dentro y fuera de la institución ${ }^{15}$.

En estos momentos de cambio, rodeados de incertidumbres y con algunas debilidades notables, parte de nuestros deberes profesionales deben dedicarse al tejido de acuerdos que permitan actuar coordinadamente en direcciones determinadas. El asociacionismo y la cooperación no son terrenos de intercambio placentero de conocimientos para cuando se termina la jornada laboral, son terrenos de definición de políticas de información, políticas sin las cuales nuestra instituciones no podrán cumplir sus finalidades.

\section{Notas}

1. "Conclusions de les Primeres jornades de teledocumentació a Catalunya". Butlletí de l'Associació de Bibliotecaris de Catalunya, 1983, n. 4, pp. 52-54.

http://www.raco.cat/index.php/ButlletiBibliotecaris/ article/view/52887/60834

2. Rose, John B. (1989). "The Unesco general information programme and its role in the development of regional cooperative networks". Proceedings of the latul conferences. Paper 6.

http://docs.lib.purdue.edu/iatul/1989/papers/6

3. Fesabid (2013). El valor económico y social de los servicios de información: bibliotecas: Informe de resultados, p. 35. http://www.fesabid.org/system/files/repositorio/fesabidvalor-economico-social-servicios-informacion-bibliotecas.pdf

4. Ver la recopilación de estudios en: Dempsey, Lorcan; Lavoie, Brian; Malpas, Constance. Understanding the collective collection: Towards a system-wide perspective on library print collections. Dublin, OH: OCLC Research, 2013. http://oclc.org/research/publications/library/2013/2013ogr.html

5. Finch, Janet (2012). “Gobiernos, empresas, organizaciones benéficas y otros invierten grandes sumas de dinero en la investigación con el fin de alcanzar estos beneficios: aumentar el conocimiento y la comprensión, y también para hacer contribuciones tangibles al bienestar social y al crecimiento económico. Para la investigación y sus productos no son sólo bienes económicos: contribuyen inconmensura- 
blemente a la vida intelectual y cultural de la nación. Por lo tanto, los gobiernos de todo el mundo ven la vitalidad de la base de investigación como algo fundamental para la salud de la economía y la sociedad modernas". Janet Finch (Chair) / Accessibility, sustainability, excellence: how to expand access to research publications: Report of the Working Group on Expanding Access to Published Research. Findings, 2012. http://apo.org.au/sites/default/files/Finch-Group-reportFINAL-VERSION.pdf

6. Björk, Bo-Christer; Welling, Patrick; Laakso, Mikael et al. (2010). "Open access to the scientific journal literature: situation 2009". En Scalas, Enrico. PLoS one, v. 5, n. 6, e11273. http://dx.doi.org/10.1371/journal.pone.0011273

7. Erway, Ricky (2013). Starting the conversation: universitywide research data management policy. Dublin, Ohio: OCLC Research.

http://www.oclc.org/content/dam/research/publications/ library/2013/2013-08.pdf

8. Fenoll, Carme (2013). "Políticas de préstamo digital en las bibliotecas públicas: el caso de Inglaterra”. Blok de BiD, 15/05/2013. http://www.ub.edu/blokdebid/es/content/pol\%C3\%ADticasde-pr\%C3\%A9stamo-digital-en-las-bibliotecasp\%C3\%BAblicas-el-caso-de-inglaterra

9. Recomiendo la lectura de los excelentes informes para el Blok de BiD hechos por Carme Fenoll y por Cristóbal Urbano sobre el préstamo de libros electrónicos en Estados Unidos, Francia e Inglaterra.

10. Quizá deberíamos hacer una excepción con las directri- ces que emanan de la Comisión Europea que en el ámbito de la investigación científica están mostrando ser rupturistas, decididas y forzadoras de consenso.

11. Fukuyama, Francis (1995). The social virtues and the creation of prosperity. Londres: Hamosh Hamilton. Versión española: Trust: la confianza. Barcelona: Ediciones B, 1998.

12. Sus inicios están explicados en: Cabo, Mercè; Celestino, Sonsoles; Guerra, Carmen; Taladriz-Mas, Margarita (2003). "Un puente hacia el futuro: el Plan estratégico de Rebiun". BiD: textos universitaris de biblioteconomia i documentació, juny, n. 10.

http://bid.ub.edu/10cabo2.htm

13. http://www.mcu.es/bibliotecas/docs/MC/ConsejoCb/ pleno/PlanEstrategicoCCBweb.pdf

14. Gallo-León, José-Pablo (coord.) (2014). Prospectiva 2020: Las diez áreas que más van a cambiar en nuestras bibliotecas en los próximos años. Consejo de Cooperación Bibliotecaria, Ministerio de Cultura.

http://www.mcu.es/bibliotecas/docs/MC/ConsejoCb/ GruposTrabajo/GE_prospectiva/Estudioprospectiva2020.pdf

Ver un resumen en:

Marquina, Julián (2014). "Transformación física y social de la biblioteca en los próximos años". JulianMarquina.es, 6 marzo. http://www.julianmarquina.es/transformacion-fisica-ysocial-de-la-biblioteca-en-los-proximos-anos

15. Esta visión sintética se la debo a Alfonso Stinus, de Momentum.

\section{Directorio EXIT \\ (EXpertos en Tratamiento de la Información) http://directorioexit.info}

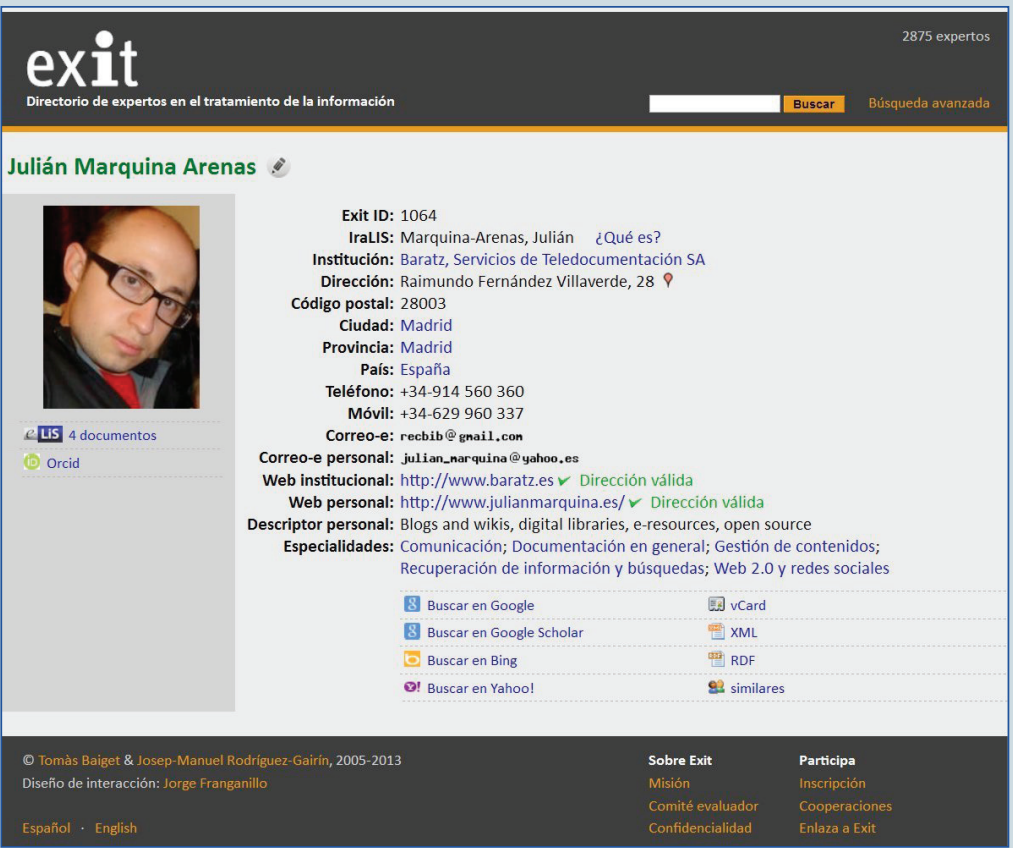

La nueva versión localiza la dirección postal en Google maps y permite buscar perfiles de expertos similares a uno dado. 


\section{Colección de libros de bolsillo}

\section{El profesional de la información (Editorial UOC)}
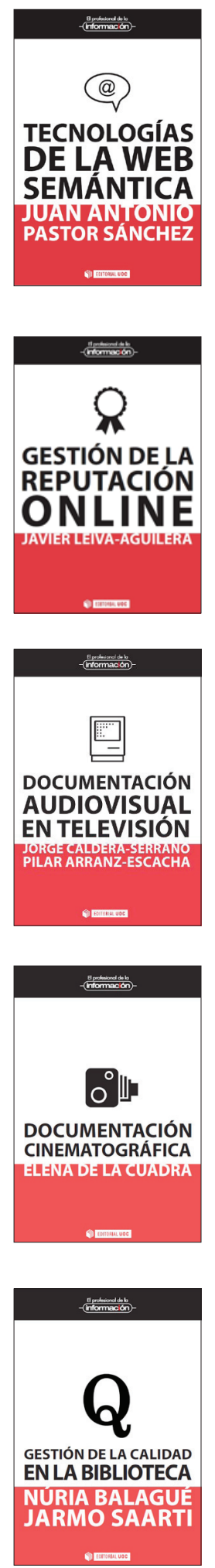
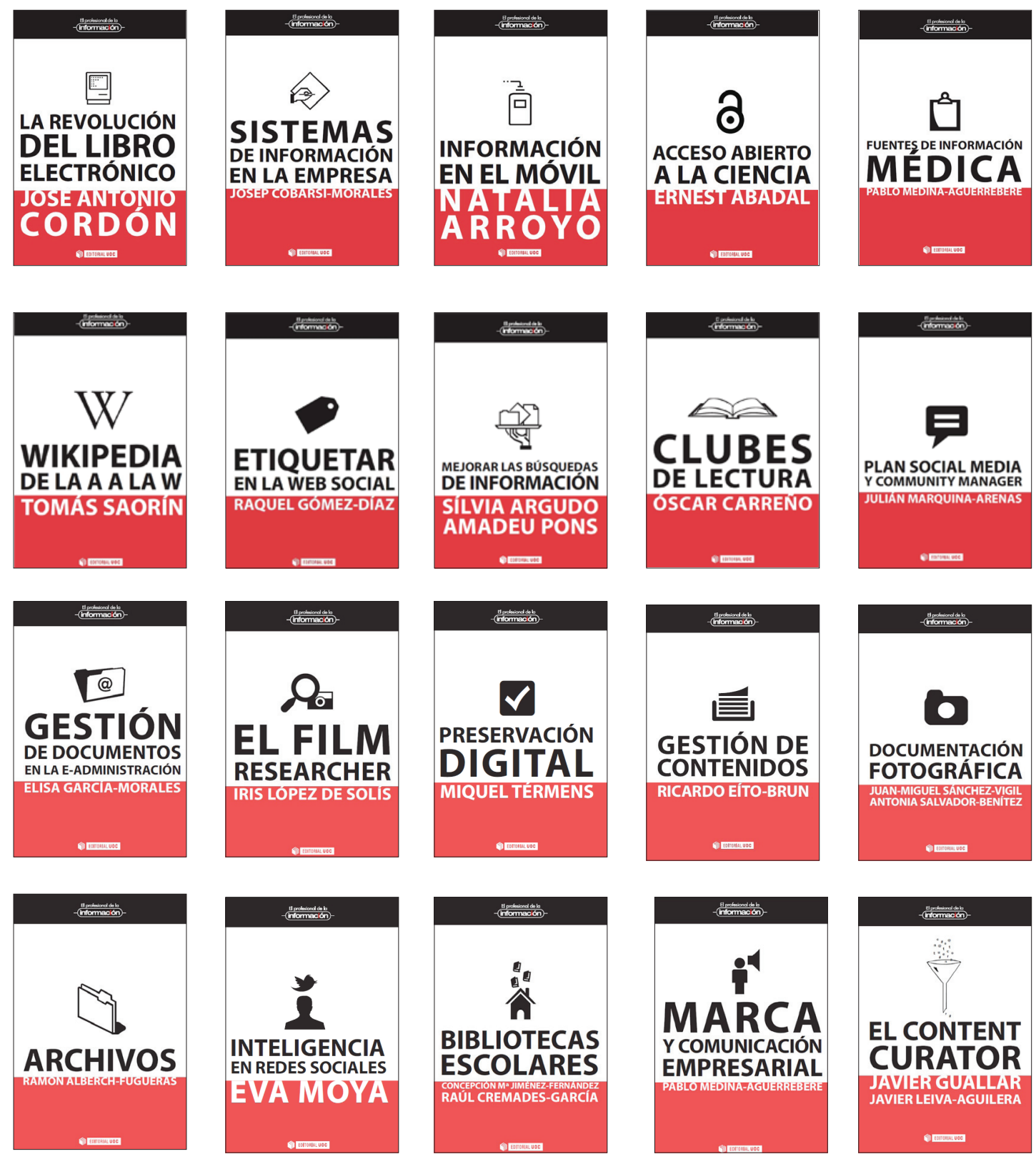

Más información:

http://www.elprofesionaldelainformacion.com/libros.html 